\title{
Solvable groups whose character degree graphs generalize squares
}

\author{
Mark L. Lewis and Qingyun Meng \\ Communicated by Robert M. Guralnick
}

\begin{abstract}
Let $G$ be a solvable group, and let $\Delta(G)$ be the character degree graph of $G$. In this paper, we generalize the definition of a square graph to graphs that are block squares. We show that if $G$ is a solvable group so that $\Delta(G)$ is a block square, then $G$ has at most two normal nonabelian Sylow subgroups. Furthermore, we show that when $G$ is a solvable group that has two normal nonabelian Sylow subgroups and $\Delta(G)$ is block square, then $G$ is a direct product of subgroups having disconnected character degree graphs.
\end{abstract}

\section{Introduction}

Throughout this paper, $G$ will be a finite group, and $\operatorname{cd}(G)=\{\chi(1) \mid \chi \in \operatorname{Irr}(G)\}$ will be the set of irreducible character degrees of $G$. The idea of studying the influence of $\operatorname{cd}(G)$ on the structure of $G$ via graphs related to $\operatorname{cd}(G)$ was introduced in [9]. The prime divisor character degree graph which is denoted by $\Delta(G)$ for $G$. We will often shorten the name of this graph to the character degree graph. The vertex set for $\Delta(G)$ is $\rho(G)$, the set of all the prime divisors of $\operatorname{cd}(G)$, and there is an edge between two vertices $p$ and $q$ if $p q$ divides some degree $a \in \operatorname{cd}(G)$.

The character degree graph has proven to be a useful tool to study the normal structure of finite solvable groups when given information about $\operatorname{cd}(G)$. For example, the first author classifies the solvable groups whose character degree graphs are disconnected in [4]. We say that the group $G$ is disconnected when $\Delta(G)$ is disconnected. In both [2] and [13], structural information is obtained for solvable groups whose character degree graphs have diameter 3. In [10], C. P. Morresi Zuccari shows if $G$ is a solvable group whose Fitting subgroup is abelian and $\Delta(G)$ has no vertex adjacent to all of the other vertices in the graph, then $G$ is a direct product of disconnected subgroups.

In [7], we prove for a solvable group $G$ that if $\Delta(G)$ is a square, then $G$ is a direct product of two subgroups of $G$ that each have disconnected graphs con-

This work was supported by China Scholarship Council (CSC) and National Science Foundation Council (NSFC) (Grant Nos. 11601121, 11771356) and Science Foundation of Henan University of Technology (Grant No. 31490036).

Corresponding author is Qingyun Meng. 
taining two vertices. A natural way to generalize this graph is to replace each vertex by a set of vertices. With this in mind, a (simple) graph is said to be a block square if its vertex set can be written as a union of four disjoint, nonempty subsets $\pi_{1}, \pi_{2}, \pi_{3}, \pi_{4}$, where no prime in $\pi_{1}$ is adjacent to any prime in $\pi_{3}$ and no prime in $\pi_{2}$ is adjacent to any prime in $\pi_{4}$, and there exist vertices in both $\pi_{1}$ and $\pi_{3}$ that are adjacent to vertices in $\pi_{2}$ and in $\pi_{4}$.

Notice that if $H$ and $K$ are disconnected groups whose graphs each have two connected components and $\rho(H) \cap \rho(K)=\varnothing$, then $\Delta(H \times K)$ will be a block square. The question arises: are there other ways to obtain a block square graph with a solvable group, or can we generalize our result in [7] from squares to block squares. We are unable at this time to fully answer this question. However, we do obtain a partial answer.

First, we see using [4] that if $G$ is solvable and disconnected, then $G$ has at most one nonabelian normal Sylow subgroup. Hence, if $G=H \times K$, where $H$ and $K$ are solvable and disconnected, then $G$ can have at most two nonabelian normal Sylow subgroups. Hence, our first result can be considered one small indication that being block square implies the direct product property.

Theorem 1.1. If $G$ is a solvable group, where $\Delta(G)$ is a block square, then $G$ has at most two normal nonabelian Sylow subgroups.

Second, there are two situations where we are able to prove that $G$ is a direct product of groups with disconnected graphs when $G$ is solvable and $\Delta(G)$ is block square. The first is when $G$ has two normal nonabelian Sylow subgroups.

Theorem 1.2. Let $G$ be a solvable group, where $\Delta(G)$ is a block square. If $G$ has two normal nonabelian Sylow subgroups, then $G=M \times N$, where $M$ and $N$ are disconnected subgroups of $G$ that satisfy $\rho(M) \cap \rho(N)=\varnothing$.

The second is the case where $G$ has one normal Sylow subgroup and the block containing the prime for this normal subgroup and the adjacent block are all single points.

Theorem 1.3. Let $G$ be a solvable group with a normal nonabelian Sylow $p$-subgroup $P$. Suppose $\Delta(G)$ is a block square whose vertex set is $\rho(G)=\{p, r, s\} \cup \pi$, where $p$ is adjacent to both $r$ and s but not adjacent to any prime in $\pi$ and both $r$ and $s$ are both adjacent to at least one vertex in $\pi$ but $r$ is not adjacent to $s$. Then $G=A \times B$, where $\rho(A)=\{p\} \cup \pi$ and $\rho(B)=\{r, s\}$.

In this paper, we study the normal structure of a finite solvable group $G$ whose character degree graph $\Delta(G)$ is a block square. At this time, it is still an open question as to whether or not $G$ must be a direct product of disconnected groups 
when $G$ is solvable, where $\Delta(G)$ is block square and $G$ has no nonabelian normal Sylow subgroups or one normal nonabelian Sylow subgroup and $\Delta(G)$ is not the particular structure studied in Theorem 1.3.

\section{Preliminaries}

We start by establishing some notation which will be used repeatedly. Given an integer $n$, define $\pi(n)$ to be the set of all the primes dividing $n$. When $G$ is a group and $H \leq G$, we write $\pi(G: H)$ for $\pi(|G: H|)$. Let $N$ be a normal subgroup of $G$. Use $\mathrm{NL}(N)$ to denote the set of nonlinear irreducible characters of $N$. For a character $\theta \in \operatorname{Irr}(N)$, we define $\operatorname{Irr}(G \mid \theta)$ for the set of irreducible constituents of $\theta^{G}$ and $\operatorname{cd}(G \mid \theta)=\{\chi(1) \mid \chi \in \operatorname{Irr}(G \mid \theta)\}$. We set

$$
\rho_{0}(G)=\left\{p \in \rho(G) \mid \text { there exists } P \in \operatorname{Syl}_{p}(G), P \triangleleft G\right\} .
$$

For other notation on group theory, the reader is referred to [3].

We remind the reader that there have been a large number of results proved about character degree graphs. A nice expository article with references covering many of these results is [6]. One result that we need is Pálfy's condition (see [12]) which states that if $G$ is solvable and $\{u, v, w\}$ are vertices in $\Delta(G)$, then there must be an edge incident to two of $\{u, v, w\}$. One consequence of this is a theorem of Manz and Wolf which is that if $G$ is solvable, then $\Delta(G)$ has diameter at most 3. We note that Pálfy's condition implies that if $G$ is solvable group so that $\Delta(G)$ is a block square, then each $\pi_{i}$ induces a complete subgraph of $\Delta(G)$.

The next lemma is useful to evaluate the cardinality of $\rho_{0}(G)$ in terms of the structure of $\Delta(G)$. It is proved as [11, Lemma 3.2], so we do not prove it here.

Lemma 2.1. Let $G$ be a solvable group. Given a prime $q \in \rho(G)$, set $\delta(q)$ to be the set of all vertices in $\rho(G)$ that are different from $q$ and not adjacent to $q$ in $\Delta(G)$. Then $\left|\rho_{0}(G) \cap \delta(q)\right| \leq 1$.

We translate this lemma to the situation where $\Delta(G)$ is a block square. Note that this is Theorem 1.1 in the introduction.

Theorem 2.2. Let $G$ be a solvable group, and assume that $\Delta(G)$ is a block square. Then $\left|\pi_{i} \cap \rho_{0}(G)\right| \leq 1, i=1, \ldots, 4$, and in particular, $\left|\rho_{0}(G)\right| \leq 2$.

Proof. Observe for each $i$ that there is some vertex of $\Delta(G)$ that is not adjacent to all of the vertices in $\pi_{i}$. Hence, we may apply Lemma 2.1 to each set $\pi_{i}$, and we have $\left|\pi_{i} \cap \rho_{0}(G)\right| \leq 1, i=1, \ldots, 4$. On the other hand, the vertices in $\rho_{0}(G)$ induce a complete subgraph of $\Delta(G)$. Observe that a complete subgraph of $\Delta(G)$ can contain vertices from at most two $\pi_{i}$. Thus, there are at most two $\pi_{i}$ having vertices in $\rho_{0}(G)$, and the result follows. 
Sometimes, when dealing with subgroups and quotients of a solvable group whose character degree graph is block square, one will encounter solvable groups whose character degree graphs are disconnected, 1-connected, or have diameter 3. We will use the following properties of $G$ when $\Delta(G)$ has one of these structures. The first result is proved as [4, Main Theorem and Lemmas 3.1-3.6].

Lemma 2.3. If $G$ is a solvable group so that $\Delta(G)$ is disconnected, then the following are true.

(a) G satisfies the hypotheses of one of [4, Examples 2.1-2.6, Section 2].

(b) $\left|\rho_{0}(G)\right| \leq 1$, and $\left|\rho_{0}(G)\right|=1$ if and only if $G$ satisfies the hypotheses of [4, Example 2.1 or 2.6].

(c) The Fitting subgroup of $G$ is a direct product of a $t$-subgroup with a central subgroup of $G$ for some prime $t$. If $G$ does not satisfy the hypotheses of [4, Example 2.4], then $t \in \rho(G)$. If $G$ does satisfy the hypotheses of [4, Example 2.2, 2.3 or 2.4], then the noncentral t-subgroup of the Fitting subgroup is minimal normal in $G$.

(d) If $G$ satisfies the hypotheses of [4, Example 2.5], then the Fitting subgroup of $G$ has a nonabelian Sylow 2-subgroup.

(e) If $G$ satisfies the hypotheses of [4, Example 2.2 or 2.3], then $\rho(G)=\{2,3\}$.

(f) If $G$ satisfies the hypotheses of [4, Example 2.1 or 2.5], then one connected component of $\Delta(G)$ consists of a single prime.

(g) Suppose $G$ satisfies the hypotheses of [4, Example 2.4]. If $F$ is the Fitting subgroup of $G$ and $E / F$ is the Fitting subgroup of $G / F$, then $G / E$ is abelian and $E / F$ is a Hall subgroup of $G / F$. Also, there exist subgroups $H$ and $V$ so that $G=V \rtimes H$, where $V$ is an elementary abelian q-group for some prime $q$, and if $K$ is the Fitting subgroup of $H$, then $V$ is irreducible as a module for $K$. If $Z=C_{H}(V)$, then the primes dividing $|K: Z|$ make up one connected component of $\Delta(G)$ and the primes that divide $|H: K|$ make up the other connected component of $\Delta(G)$. Finally, $V=[E, F]$

(h) If $G$ satisfies the hypotheses of [4, Example 2.1], then $\rho_{0}(G)$ is a connected component of $\Delta(G)$. If $G$ satisfies the hypotheses of [4, Example 2.6], then the connected component of $\Delta(G)$ containing $\rho_{0}(G)$ has at least one additional vertex, and thus, $\rho(G)$ contains at least 3 primes.

(i) $G$ has Fitting height 2 if and only if $G$ satisfies the hypotheses of [4, Example 2.1].

Given the length of the descriptions of [4, Examples 2.1-2.6], we do not repeat those descriptions here, but we refer the reader to that paper. When a group has 
a disconnected degree graph, we will refer to these examples throughout the paper with reference to [4].

This next result is proved as [13, Theorem 3] or [2, Theorem A].

Lemma 2.4. Let $G$ be a solvable group with $\Delta(G)$ having diameter 3 . Then we have $\left|\rho_{0}(G)\right|=1$.

A cut vertex of a graph is a vertex of the graph, where the induced graph by all of the rest vertices of the graph has more connected components than the original graph. A graph $\Delta$ is said to be 1-connected if it is connected and contains a cut vertex. The following is [8, Theorem 2.2].

Lemma 2.5. If $G$ is a solvable group such that $\Delta(G)$ is 1-connected and $G$ has two nonabelian normal Sylow subgroups, then $G$ has a normal nonabelian Sylow $p$-subgroup, where $p$ is the prime associated with the cut vertex in $\Delta(G)$ and $G=P \times H$, where $H$ is the Hall $p$-complement of $G$ and $H$ has a unique normal nonabelian Sylow subgroup.

Let $G$ be a group. Following Morresi Zuccari in [11], we define $\Sigma(G)$ to be the primes in $\rho(G)$ that are not adjacent to some prime in $\rho_{0}(G)$ in $\Delta(G)$. This next lemma is [11, Lemma 2.3].

Lemma 2.6. Let $G$ be solvable with $F=\mathrm{F}(G)$, and suppose that $\Sigma(G)$ is not empty. Then the following are true.

(a) The $\Sigma(G)$-Hall subgroups are abelian.

(b) If there exist primes $p \in \rho_{0}(G)$ and $q \in \Sigma(G)$ so that $p$ is not adjacent to $q$, and $K$ is a p-complement in $G$, then there exists a subgroup $Q \in \operatorname{Syl}_{q}(G)$ such that $Q \leq \mathrm{C}_{K}\left(O_{p^{\prime}}(F)\right)$.

Next consider solvable groups with two normal nonabelian Sylow subgroups.

Lemma 2.7. Let $G$ be solvable with two normal nonabelian Sylow subgroups: $P$ is a Sylow $p$-subgroup and $Q$ is a Sylow $q$-subgroup for distinct primes $p$ and $q$. Then $\rho\left(G /\left(P^{\prime} Q^{\prime}\right)\right)=\rho(G) \backslash\{p, q\}$.

Proof. Clearly, $\rho\left(G /\left(P^{\prime} Q^{\prime}\right)\right) \subseteq \rho(G) \backslash\{p, q\}$ by Itô's theorem since $G / P^{\prime} Q^{\prime}$ will have normal abelian Sylow $p$ - and Sylow $q$-subgroups. Suppose

$$
r \in \rho(G) \backslash \rho\left(G /\left(P^{\prime} Q^{\prime}\right)\right) .
$$

Let $R$ be a Sylow $r$-subgroup of $G$. Then, by the Itô-Michler theorem, we know that $G /\left(P^{\prime} Q^{\prime}\right)$ has a normal abelian Sylow $r$-subgroup $R P^{\prime} Q^{\prime} /\left(P^{\prime} Q^{\prime}\right)$. In particular, when $r$ is not $p$ or $q$, we deduce that $R$ is abelian and $R P^{\prime} Q^{\prime} \unlhd G$. Applying the Frattini argument, we obtain $G=R P^{\prime} Q^{\prime} N_{G}(R)$. Notice that $R \leq N_{G}(R)$. 
Since $P^{\prime} Q^{\prime} \leq \Phi(G)$, it follows that $G=N_{G}(R)$, and thus, $R \triangleleft G$. Now, $G$ has a normal abelian Sylow $r$-subgroup $R$. This is a contradiction since we assumed $r \in \rho(G)$. Therefore, no such prime $r$ exists, and we conclude that

$$
\rho\left(G /\left(P^{\prime} Q^{\prime}\right)\right)=\rho(G) \backslash\{p, q\}
$$

as desired.

We next have another result due to Morresi Zuccari. In particular, he looks at groups where the degree graph contains no vertex that is adjacent to all the others. This result is proved as [10, Theorem A].

Lemma 2.8. Let $G$ be solvable with $F=\mathrm{F}(G)$ abelian. Suppose that the degree in $\Delta(G)$ of every $v \in \rho(G)$ is at most $|\rho(G)|-2$. Then, for an integer $n$, $F=M_{1} \times \cdots \times M_{n} \times Z$ with $M_{1}, \ldots, M_{n}$ minimal normal subgroups of $G$ and $Z=Z(G)$; moreover, $G=D_{1} \times \cdots \times D_{n}$, where $M_{1} \leq D_{1}, \ldots, M_{n} \leq D_{n}$, $\left(\left|D_{i} / M_{i}\right|,\left|D_{j} / M_{j}\right|\right)=1$ for every $i \neq j$, and $\Delta\left(D_{1}\right), \ldots, \Delta\left(D_{n}\right)$ are disconnected graphs.

We conclude this section by showing that if $G$ is a solvable group, where $\Delta(G)$ is a block square $G=M \times N$ with $M$ and $N$ nonabelian, then $\Delta(M)$ and $\Delta(N)$ are disconnected.

Lemma 2.9. Let $G$ be a solvable group, where $\Delta(G)$ is a block square. If there exist nonabelian subgroups $M$ and $N$ of $G$ so that $G=M \times N$, then $\Delta(M)$ and $\Delta(N)$ are disconnected and $\rho(M) \cap \rho(N)=\varnothing$.

Proof. We begin by noting that every prime in $\rho(M)$ is adjacent to every prime in $\rho(N)$. Thus, any prime in $\rho(M) \cap \rho(N)$ will be adjacent to all of the primes in $\rho(M) \cup \rho(N)=\rho(G)$. The hypothesis that $\Delta(G)$ is block square implies that there is no prime in $\rho(G)$ that is adjacent to all of the primes in $\rho(G)$. Thus, we must have $\rho(M) \cap \rho(N)=\varnothing$. Without loss of generality, we may assume $\rho(M) \cap \pi_{1} \neq \varnothing$. Since every prime in $\rho(N)$ is adjacent in $\Delta(G)$ to every prime in $\rho(M)$, we see that $\rho(N) \cap \pi_{3}=\varnothing$. Since

$$
\rho(G)=\rho(M) \cup \rho(N) \text { and } \rho(M) \cap \rho(N)=\varnothing,
$$

we must have $\pi_{3} \subseteq \rho(M)$. Using the same reasoning, we obtain $\pi_{1} \cap \rho(N)=\varnothing$, and so $\pi_{1} \subseteq \rho(M)$.

On the other hand, recall that $N$ is nonabelian and

$$
\rho(G)=\pi_{1} \cup \pi_{2} \cup \pi_{3} \cup \pi_{4}=\rho(M) \cup \rho(N) .
$$

Observe that $\rho(N) \subseteq \pi_{2} \cup \pi_{4}$. Without loss of generality, we may assume that $\rho(N) \cap \pi_{2} \neq \varnothing$. It follows that $\pi_{4} \cap \rho(M)=\varnothing$, and so $\pi_{4} \subseteq \rho(N)$. Further- 
more, this implies that $\pi_{2} \subseteq \rho(N)$. We conclude that

$$
\rho(M)=\pi_{1} \cup \pi_{3} \text { and } \rho(N)=\pi_{2} \cup \pi_{4},
$$

and the result is proved.

\section{Proof of Theorem 1.2}

We are now ready to prove Theorem 1.2 . We begin with the special case where $\left|\pi_{1}\right|=1$.

Theorem 3.1. Let $G$ be solvable with two normal nonabelian Sylow subgroups: Sylow p-subgroup $P$ and Sylow $q$-subgroup $Q$ for distinct primes $p$ and $q$. If $\Delta(G)$ is a block square with $\pi_{1}=\{p\}$, then $G=M \times N$, where $M$ and $N$ are Hall subgroups of $G$ and $\Delta(M)$ and $\Delta(N)$ are disconnected with $\rho(N)=\pi_{1} \cup \pi_{3}$ and $\rho(M)=\pi_{2} \cup \pi_{4}$.

Proof. We prove this in a series of claims. Write $G=P \rtimes H$, where $H$ is a Hall $p$-complement of $G$. Since $q$ must be adjacent to $p$, we see that $q \in \pi_{2} \cup \pi_{4}$. Without loss of generality, we may assume that $q \in \pi_{2}$.

Claim 1. There exists a character $\theta \in \mathrm{NL}(P)$ that extends to $G$.

Proof. Since $P$ and $H$ have coprime orders, to prove Claim 1, it is sufficient to show that there exists a character $\theta \in \mathrm{NL}(P)$ such that $C_{H}(\theta)=H$. We suppose that every character $\vartheta \in \mathrm{NL}(P)$ satisfies $C_{H}(\vartheta)<H$. Consider a character $\vartheta \in \mathrm{NL}(P)$. It is easy to see that the stabilizer $I$ of $\vartheta$ in $G$ is $I=P C_{H}(\vartheta)$. Using Clifford's theorem, we see that $|G: I|=\left|H: C_{H}(\vartheta)\right|$ divides the degree of every character in $\operatorname{Irr}(G \mid \vartheta)$. Hence, every prime in $\pi\left(H: C_{H}(\vartheta)\right)$ is adjacent to $p$ in $\Delta(G)$. This implies that $\pi\left(H: C_{H}(\vartheta)\right) \subseteq \pi_{2} \cup \pi_{4}$ since $p$ is not adjacent to any primes in $\pi_{3}$.

Suppose there is a character $\theta \in \mathrm{NL}(P)$ such that $\pi_{4} \cap \pi\left(H: C_{H}(\theta)\right) \neq \varnothing$. Say $r \in \pi_{4} \cap \pi\left(H: C_{H}(\theta)\right)$. Since $Q$ centralizes $P$, we see that $Q \unlhd C_{H}(\theta)$. Since $Q$ is nonabelian, this implies that $q \in \rho\left(C_{H}(\theta)\right)$. Applying Gallagher's theorem, we see that $\operatorname{Irr}(I \mid \theta)$ will contain a character whose degree is divisible by $p q$. Using Clifford's theorem, we obtain a character $\chi \in \operatorname{Irr}(G \mid \theta)$ such that $p q r \mid \chi(1)$, violating the hypothesis that $q$ is not adjacent to any prime in $\pi_{4}$. Hence, for any character $\theta \in \mathrm{NL}(P)$, we must have $\pi\left(H: C_{H}(\theta)\right) \subseteq \pi_{2}$.

This implies that any irreducible character in $\operatorname{Irr}(G)$ whose degree is divisible by $p$ also has degree divisible by some prime in $\pi_{2}$. Since no prime in $\pi_{4}$ can be adjacent to a prime in $\pi_{2}$, we deduce that no character in $\operatorname{Irr}(G)$ whose degree is divisible by $p$ is divisible by a prime in $\pi_{4}$. In particular, we conclude that $p$ is not adjacent to any prime in $\pi_{4}$, which is a contradiction. 
Claim 2. $\Delta(H)$ is disconnected with two connected components $\rho(H) \cap \pi_{2}$ and $\rho(H) \cap \pi_{4}$.

Proof. By Claim 1, we know that there is a character $\theta \in \mathrm{NL}(P)$ that extends to $G$. Applying Gallagher's theorem, we determine that $\rho(H) \cap \pi_{3}=\varnothing$. Since $q \in \rho(H)$, we see that $\rho(H) \cap \pi_{2} \neq \varnothing$. Suppose $\rho(H) \cap \pi_{4}=\varnothing$; then $H$ has normal abelian Hall $\pi_{3} \cup \pi_{4}$-subgroup, which we denote by $Y$. Write $X$ for the Hall $\pi_{4}$-subgroup of $Y$. Then $P X$ is normal in $G$. Since $p$ is adjacent to a prime in $\pi_{4}$ in $\Delta(G)$ and $|G: P X|$ is not divisible by any prime in $\{p\} \cup \pi_{4}$, there exists a character $\tau \in \mathrm{NL}(P X)$ so that $\tau(1)$ is divisible by $p$ and some prime in $\pi_{4}$. Let $\psi$ be an irreducible constituent of $\tau^{G}$. We know that $\psi(1) / \tau(1)$ divides $|G: P X|$. Note that the only primes that divide $\psi(1)$ must lie in $\{p\} \cup \pi_{4}$, and since no prime in $\{p\} \cup \pi_{4}$ divides $|G: P X|$, we conclude that $\psi(1)=\tau(1)$. Thus, every character in $\operatorname{Irr}(G \mid \tau)$ is extension of $\tau$. In light of Gallagher's theorem, we have that $H / X$ is abelian. However, $Q \cap X=1$ and $Q \leq H$, so $Q \cong Q X / Q \leq H / X$ which is a contradiction since $Q$ is nonabelian. Therefore, $\rho(H) \cap \pi_{4} \neq \varnothing$. Notice that $H \cong G / P$, so $\Delta(H)$ is a subgraph of $\Delta(G)$. We conclude that $\Delta(H)$ is disconnected with two connected components: $\rho(H) \cap \pi_{2}$ and $\rho(H) \cap \pi_{4}$.

Claim 3. $G=M \times N$, where $M$ and $N$ are the Hall $\{p\} \cup \pi_{3} \cup \rho(G)^{\prime}$-subgroup and the Hall $\pi_{2} \cup \pi_{4}$-subgroup of $G$, respectively.

Proof. Let $A$ be the Hall $\rho(G)^{\prime}$-subgroup of $G$, let $W$ be the Hall $\pi_{3}$-subgroup of $H$, and let $U$ be a Hall $\pi_{2} \cup \pi_{4}$-subgroup of $H$. By Claim 2, we know that $H=(A \times W) \rtimes U$ is a solvable group so that $\Delta(H)$ is disconnected. Since $H$ has normal nonabelian Sylow $q$-subgroup $Q$, we know from Lemma 2.3 (b) that $H$ satisfies the hypotheses of [4, Example 2.1 or 2.6]. Using Lemma 2.3 (c) with respect to $H$, we see that $A \times W \leq Z(H)$ and $H=A \times W \times U$. It follows that $\Delta(H)=\Delta(U)$.

Writing $L=P U$, we consider $L / P^{\prime}$. It is not difficult to see that $\Delta\left(L / P^{\prime}\right)$ is disconnected. Observe that $P / P^{\prime}$ will be a normal $p$-subgroup of $L / P^{\prime}$. Since $Q P^{\prime} / P^{\prime}$ is a noncentral subgroup of $L / P^{\prime}$, we may use Lemma 2.3 (c) to see that $P / P^{\prime}$ is a central subgroup of $L / P^{\prime}$. It follows that $L / P^{\prime}=P / P^{\prime} \times U P^{\prime} / P^{\prime}$. In particular, $P / P^{\prime} \leq Z\left(L / P^{\prime}\right)$ and $U$ acts trivially on $P / P^{\prime}$. Since $P^{\prime} \leq \Phi(P)$, we obtain $L=P \times U$. We set $M=P \rtimes(W \times A)$. It is clear that $G=M \times U$, where $\Delta(M)$ and $\Delta(U)$ are disconnected. (For the record, $M$ satisfies the hypotheses of [4, Example 2.1], and $U$ satisfies the hypotheses of [4, Example 2.1 or 2.6].)

This gives the desired conclusion. 
This next theorem includes most of the work in proving Theorem 1.2.

Theorem 3.2. Let $G$ be a solvable group with two normal nonabelian Sylow subgroups: $P$ a Sylow $p$-subgroup and $Q$ a Sylow $q$-subgroup for distinct primes $p$ and $q$. If $\Delta(G)$ is block square with $p \in \pi_{1}, q \in \pi_{2}$ and $\left|\pi_{i}\right| \geq 2, i=1,2$, then $G=M \times N$, where $\Delta(M)$ and $\Delta(N)$ are disconnected with $\rho(M)=\pi_{1} \cup \pi_{3}$ and $\rho(N)=\pi_{2} \cup \pi_{4}$ and $P \leq M$ and $Q \leq N$.

Proof. Let $G$ be a counterexample with $|G|$ minimal.

Claim 1. $P^{\prime}$ and $Q^{\prime}$ are minimal normal subgroups of $G$.

Proof. Suppose that $P_{1}$ is a normal subgroup of $G$ that is properly contained in $P^{\prime}$. Consider factor group $G / P_{1}$. Since $\rho\left(G / P^{\prime}\right)=\rho(G) \backslash\{p\}$ (see [5, Lemma 3]) and $P / P_{1}$ will be a nonabelian subgroup of $G / P_{1}$, we see that $\rho\left(G / P_{1}\right)=\rho(G)$. It is not difficult to see that any normal Sylow subgroup of $G$ will produce a normal Sylow subgroup of $G / P_{1}$. Conversely, let $R$ be a Sylow $r$-subgroup of $G$ for some prime $r$ that is distinct from $p$, and suppose that $R P_{1} / P_{1}$ is normal in $G / P_{1}$. This implies that $R P_{1} / P_{1}$ centralizes $P / P_{1}$. Hence, $R$ will centralize $P / P_{1}$. Since $P_{1} \leq P^{\prime} \leq \Phi(P)$, we conclude that $R$ centralizes $P$. Now, $R$ centralizes $P_{1}$, so $R$ is normal in $R P_{1}$. This implies that $R$ is characteristic in $R P_{1}$, so $R$ is normal in $G$. Notice that $R \cong R P_{1} / P_{1}$. We conclude that $\rho_{0}\left(G / P_{1}\right)=\rho_{0}(G)$, and $\left|\rho_{0}\left(G / P_{1}\right)\right|=\left|\rho_{0}(G)\right|=2$.

By Lemmas 2.3 and 2.4 , we know if $\Delta\left(G / P_{1}\right)$ were disconnected or diameter 3, then $\left|\rho_{0}\left(G / P_{1}\right)\right| \leq 1$. Since this is not the case, we must have that $\Delta\left(G / P_{1}\right)$ is block square. If $P_{1}>1$, then we may use the induction hypothesis to see that $G / P_{1}$ is a direct product of two subgroups whose graphs are disconnected; say $G / P_{1}=M / P_{1} \times L / P_{1}$, where $\Delta\left(M / P_{1}\right)$ has the two components $\pi_{1}$ and $\pi_{3}$ and $\Delta\left(L / P_{1}\right)$ has $\pi_{2}$ and $\pi_{4}$ as its two components and $P \leq M$ and $Q \leq L$. Since both of $M / P_{1}$ and $L / P_{1}$ have a normal nonabelian Sylow subgroup and each $\left|\pi_{i}\right| \geq 2$ for $i=1,2$, we also know via Lemma 2.3 (b) and (h) that $M / P_{1}$ and $L / P_{1}$ satisfy the hypotheses of [4, Example 2.6].

Since $L$ centralizes $P / P_{1}$ and $P_{1}<P^{\prime} \leq \Phi(G)$, it follows that $L$ centralizes $P$. Notice that $P_{1}$ is the Sylow $p$-subgroup of $L$ and that $P_{1}$ is central in $L$. Take $N$ be a Hall $p$-complement of $L$, and since $P_{1}$ centralizes $N$, we see that $N$ is normal $L$. This implies that $N$ is characteristic in $L$, so $N$ is normal in $G$. We also see that $N \cap M=\{1\}$, so $G=M \times N$, a contradiction. Therefore, we must have that $P^{\prime}$ is minimal normal in $G$. Using a similar argument, we conclude that $Q^{\prime}$ is a minimal normal subgroup of $G$.

Claim 2. $G$ has no central $r$-subgroup, for any prime $r \in \pi(G) \backslash\{p, q\}$. 
Proof. Suppose $Z \leq Z(G)$ is a nontrivial $r$-subgroup. Consider the factor group $G / Z$. We claim that $\rho(G / Z)=\rho(G)$. To prove this, suppose there is a prime $s \in \rho(G) \backslash \rho(G / Z)$, and let $S$ be a Sylow $s$-subgroup of $G$. Then $S Z$ is a normal subgroup of $G$. If $s=r$, then $S=S Z$, so $S$ is normal in $G$. This cannot occur since $r$ is not in $\rho_{0}(G)=\{p, q\}$. On the other hand, if $s \neq r$, then $S$ is characteristic in $S Z=S \times Z$, and since $S Z$ is normal in $G$, we obtain $S$ is normal in $G$. Since $s$ is not in $\rho(G / Z)$, we must have that $S Z / Z$ is a abelian. Since $S \cong S Z / Z$, we deduce that $S$ is abelian and normal in $G$, contradicting with $s \in \rho(G)$. This proves the claim.

Also, a similar proof proves that $\rho_{0}(G / Z)=\rho_{0}(G)$. Now, $\left|\rho_{0}(G / Z)\right|=2$, so we use Lemmas 2.3 and 2.4 to see that $\Delta(G / Z)$ is not disconnected and does not have diameter 3 . We deduce that $\Delta(G / Z)$ is connected and has diameter 2 . Hence, $\Delta(G / Z)$ is block square, too. By induction, $G / Z=M / Z \times N / Z$ for some normal subgroups $M, N \leq G$, where $\Delta(M / Z)$ has connected components $\pi_{1}, \pi_{3}$ and $\Delta(N / Z)$ has connected components $\pi_{2}, \pi_{4}$, and $P \leq M$ and $Q \leq N$. Suppose there exists a prime $s \in \rho(M) \backslash \rho(M / Z)$. Observe that $s \in \pi_{2} \cup \pi_{4}$. Suppose first that $s \neq r$, and let $S$ be a Sylow $s$-subgroup of $M$. We must have that $S Z$ is normal in $M$, so $S$ is characteristic in $S \times Z$. We deduce that $S$ is normal in $M$ and $S \cong S Z / Z$ is abelian. This contradicts $s \in \rho(M)$. Hence, $s=r$.

The result of the previous paragraph shows that $\rho(M) \subseteq \rho(M / Z) \cup\{r\}$, where $\rho(M / Z)=\pi_{1} \cup \pi_{3}$. Using a similar argument on $N$, we have

$$
\rho(N) \subseteq \rho(N / Z) \cup\{r\},
$$

where $\rho(N / Z)=\pi_{2} \cup \pi_{4}$. Since $r$ can only lie in one of $\pi_{1} \cup \pi_{3}$ and $\pi_{2} \cup \pi_{4}$, we see that $\rho(N) \supset \rho(N / Z)$ and $\rho(M) \supset \rho(M / Z)$ cannot occur simultaneously. There are three possible cases.

Case 1: $\rho(M)=\rho(M / Z)$ and $\rho(N)=\rho(N / Z)$. In this case, both $M$ and $N$ are solvable groups such that $\Delta(M)$ and $\Delta(N)$ are disconnected and both $M$ and $N$ satisfy the hypotheses of [4, Example 2.6] (this follows from Lemma 2.3 (b) and (h)). Observe that $\rho(G)=\rho(M) \cup \rho(N)$. Suppose $r \in \rho(G)$, and without loss of generality, we may assume $r \in \rho(M)$. Since $\rho(M) \cap \rho(N)=\varnothing$, we see that $r$ cannot lie in $\rho(N)$. In particular, if we write $R$ for a Sylow $r$-subgroup of $N$, then $R$ must lie in the center of $N$ by Lemma 2.3 (c). Let $H$ be a Hall $r$-complement in $N$, so $N=R \times H$. Then $G=M R \times H$, a contradiction to the choice of $G$ as a counterexample.

We now have the case where $r$ is not in $\rho(G)$. We have $M=R \times H$, and $N=S \times K$ by Lemma 2.3 (c), where $R$ and $S$ are the Sylow $r$-subgroups of $M$ and $N$, respectively, and $H$ and $K$ are the Hall $r$-complements of $M$ and $N$, respectively. Observe that $Z \leq R \cap S$ and $G=R S H \times K$, a contradiction to the choice of $G$. We conclude that this case does not occur. 
Case 2: $\rho(M)=\rho(M / Z) \cup\{r\}$ and $\rho(N)=\rho(N / Z)$, where $r \in \pi_{2} \cup \pi_{4}$. Take $R$ to be a Sylow $r$-subgroup of $M$. Notice that $R=R Z$ is normal in $M$. Now, $M$ contains two normal nonabelian Sylow subgroups $P$ and $R$. Thus, we can use Lemmas 2.3 and 2.4 to see that $\Delta(M)$ is connected and has diameter 2. Notice that $r$ will be a cut vertex for $\Delta(M)$, so $\Delta(M)$ is 1-connected. By Lemma 2.5, $M=R \times H$, where $H$ is the Hall $r$-complement of $M$. It is easy to see that $G=H \times R N$, a contradiction to the choice of $G$. This proves that this case cannot occur.

Case 3: $\rho(M)=\rho(M / Z)$ and $\rho(N)=\rho(N / Z) \cup\{r\}$, where $r \in \pi_{1} \cup \pi_{3}$. Using an argument similar to the one applied in case 2 , we may conclude that this case cannot occur.

Write $B$ for the Hall $\{p, q\}$-complement of $F$, the Fitting subgroup of $G$.

Claim 3. $B$ is abelian.

Proof. If $B=1$, then the claim is trivial. Thus, we assume that $B>1$. Let

$$
K=\mathrm{C}_{G}(Q B) \mathrm{C}_{G}(P B) B .
$$

Observe that $P \leq C_{G}(Q B)$ and $Q \leq C_{G}(P B)$, and since $B \leq F$, we have that $F \leq K$. It is easy to see that $K$ is normal in $G$. Note that no prime in $\pi_{3}$ is adjacent to $p$, so $\pi_{3} \subseteq \Sigma(G)$. Similarly, no prime in $\pi_{4}$ is adjacent to $q$, so $\pi_{4} \subseteq \Sigma(G)$. Thus, $\pi_{3} \cup \pi_{4} \subseteq \Sigma(G)$. By Lemma 2.6, we see that $G$ has abelian Hall $\pi_{3} \cup \pi_{4^{-}}$ subgroup and that $K$ contains a Hall $\pi_{3} \cup \pi_{4}$-subgroup of $G$.

We now show that $K$ is a proper subgroup of $G$. Suppose that $K=G$. Observe that $K \leq \mathrm{C}_{G}(B) B$, and this forces that $G=B C_{G}(B)$. Let $R$ be a nontrivial Sylow $r$-subgroup of $B$ for some prime $r$, and write $Z_{r}$ for the center of $R$. Since $B$ is nilpotent, we see that $Z_{r}$ is central in $B$. The fact that $G=C_{G}(B) B$ implies that $Z_{r}$ is a nontrivial central subgroup of $G$, contradicting Claim 2. Hence, $K$ is proper in $G$. It is easy to see that $\rho_{0}(K)=\{p, q\}$ and $F$ is the Fitting subgroup of $K$. Since $K$ contains a Hall $\pi_{3} \cup \pi_{4}$ subgroup of $G$, we must have $\pi_{3} \cup \pi_{4} \subseteq \rho(K)$. Once again, we may apply Lemmas 2.3 and 2.4 to see that $\Delta(M)$ is connected and has diameter 2 . This forces $\Delta(K)$ to be block square.

Using either Theorem 3.1 or the inductive hypothesis, we know that $K$ is a direct product of two solvable subgroups with disconnected character degree graphs, say $K=K_{1} \times K_{2}$. Observe that each $K_{i}$, for $i=1$, 2, contains a normal nonabelian Sylow subgroup. Hence, applying Lemma 2.3 (b), we see that each $K_{i}$ satisfies the hypotheses of [4, Example 2.1 or 2.6]. By the properties of Examples 2.1 and 2.6 found in Lemma 2.3 (c), we know $\mathrm{F}(K)=\mathrm{F}\left(K_{1}\right) \times \mathrm{F}\left(K_{2}\right)=P \times Z_{1} \times Q \times Z_{2}$, 
where $Z_{i} \leq Z\left(K_{i}\right), i=1,2$. Recalling that

$$
F=P \times Q \times B=F(K)=P \times Q \times Z_{1} \times Z_{2},
$$

it follows that $B=Z_{1} \times Z_{2}$ is abelian.

Consider the factor group $\bar{G}=G /\left(P^{\prime} Q^{\prime}\right)$. We know that $\rho(\bar{G})=\rho(G) \backslash\{p, q\}$ by Lemma 2.7. Recall that $\left|\pi_{i}\right| \geq 2$, for $i=1,2$, and $\Delta(\bar{G})$ is a subgraph of $\Delta(G)$. Hence, $\Delta(\bar{G})$ has no vertex that is adjacent to all of the other vertices in the graph. Also, observe that $\mathrm{F}(\bar{G})=F /\left(P^{\prime} \times Q^{\prime}\right)$ is abelian. Notice that neither $\bar{P}$ nor $\bar{Q}$ lies in the center of $\bar{G}$. By Lemma 2.3 (c), $\Delta(\bar{G})$ cannot be disconnected since the Fitting subgroup cannot have noncentral Sylow subgroups for two different primes.

We have that $\Delta(\bar{G})$ is connected. Notice that this implies that $\Delta(\bar{G})$ is block square. By Lemma 2.8 , there exist subgroups, say $\bar{D}$ and $\bar{E}$ and $\bar{P}^{*}$ and $\bar{Q}^{*}$, so that $\bar{G}=\bar{D} \times \bar{E}$ and $\mathrm{F}(\bar{G})=\bar{P}^{*} \times \bar{Q}^{*} \times \mathrm{Z}(\bar{G})$, where $\bar{P}^{*} \leq \bar{D}, \bar{Q}^{*} \leq \bar{E}$ and $\bar{P}^{*}$ and $\bar{Q}^{*}$ are minimal normal subgroups of $\bar{G}$ and $\Delta(\bar{D})$ and $\Delta(\bar{E})$ are disconnected. Without loss of generality, we may assume $\bar{P}^{*} \leq \bar{P}$ and $\bar{Q}^{*} \leq \bar{Q}$. In particular, $\bar{B} \leq \mathrm{Z}(\bar{G})$, so $[B, G] \leq P^{\prime} Q^{\prime} \cap B=\{1\}$. This implies that $B \leq \mathrm{Z}(G)$, and by Claim 2, we have $B=1$.

By Lemma 2.8, we also know that $\left(\left|D / P^{*}\right|,\left|E / Q^{*}\right|\right)=1$. And note that $\mathrm{F}(\bar{D})$ and $\mathrm{F}(\bar{E})$ are abelian, so $\bar{D}$ and $\bar{E}$ cannot satisfy the hypotheses of [4, Examples $2.1,2.5$ or 2.6] by Lemma 2.3 (b) and (d). From Lemma 2.3 (c), this implies that the noncentral portion of the Fitting subgroups of $\bar{D}$ and $\bar{E}$ are minimal normal subgroups, so the noncentral portion of $\mathrm{F}(\bar{D})$ is $\bar{P}^{*}$ and of $\mathrm{F}(\bar{E})$ is $\bar{Q}^{*}$. In particular, the Sylow $q$-subgroup of $\bar{D}$ lies in the center of $\bar{D}$, and the Sylow $p$-subgroup of $\bar{E}$ lies in the center of $\bar{E}$.

Write $\bar{X}$ for the Hall $q$-complement of $\bar{D}$ direct product with the Sylow $p$-subgroup of $\bar{E}$ and $\bar{Y}$ for the Hall $p$-complement of $\bar{E}$ direct product with Sylow $q$-subgroup of $\bar{D}$. Then $\bar{G}=\bar{X} \times \bar{Y}$ and $X \cap Y=P^{\prime} Q^{\prime}$. We now have $Q^{\prime}$ as the Sylow $q$-subgroup of $X$ and $P^{\prime}$ as the Sylow $p$-subgroup of $Y$. Recall that $P^{\prime}$ and $Q^{\prime}$ are normal and abelian by Claim 1 .

Suppose that $\rho(\bar{D})=\pi_{1} \cup \pi_{3} \backslash\{p\}$ and $\rho(\bar{E})=\pi_{2} \cup \pi_{4} \backslash\{q\}$. We then obtain $\rho(X)=\rho(\bar{X}) \cup\{p\}=\pi_{1} \cup \pi_{3}$ and $\rho(Y)=\rho(\bar{Y}) \cup\{q\}=\pi_{2} \cup \pi_{4}$. Writing $M$ for the Hall $q$-complement of $X$ and $N$ for the Hall $p$-complement of $Y$, then we have $G=M \times N$ as desired.

The other possibility is that $\rho(\bar{D})=\pi_{2} \backslash\{q\} \cup \pi_{4}$ and $\rho(\bar{E})=\pi_{1} \backslash\{p\} \cup \pi_{3}$. We then have $\bar{P} \times \bar{Y}$ is normal in $\bar{G}$. Notice that $Y=\left(Q \times P^{\prime}\right) \rtimes V$, where $V$ is a Hall $\pi_{1} \backslash\{p\} \cup \pi_{3}$-subgroup of $Y$. Because $[\bar{P}, \bar{Y}]=\overline{1}$, we have $[P, V]=\{1\}$. Now, $P Y=P \times(Q \rtimes V)$, and $P Y$ is normal in $G$. This implies that $p$ is adjacent to the primes in $\pi_{3}$, a contradiction. This proves the result.

We now prove Theorem 1.2. 
Proof of Theorem 1.2. We are assuming $G$ has two normal nonabelian Sylow subgroups. Say that $\rho_{0}(G)=\{p, q\}$. If one of $p$ or $q$ is the only prime in one of the $\pi_{i}$, then the result holds by Theorem 3.1. Suppose this does not happen. By Theorem 2.2, we see that $p$ and $q$ must lie in different $\pi_{i}$. Also, note that $p$ and $q$ are adjacent in $\Delta(G)$. So, without loss of generality, we may assume that $p \in \pi_{1}$, $q \in \pi_{2}$ and both $\left|\pi_{1}\right|$ and $\left|\pi_{2}\right|$ are at least 2 . Thus, we now apply Theorem 3.2 to obtain the conclusion.

\section{Proof of Theorem 1.3}

We start by stating the hypothesis that we study in this section.

Hypothesis 4.1. Let $G$ be a solvable group with a normal nonabelian Sylow p-subgroup $P$ and a p-complement $H$. Write A for the Hall $\rho(G)^{\prime}$-subgroup of $G$. Suppose $\Delta(G)$ is a block square whose vertex set is $\rho(G)=\{p, r, s\} \cup \pi$, where $\pi$ is nonempty. Assume $p$ is adjacent to both $r$ and $s$ but not adjacent to any prime in $\pi$, and $r$ and $s$ are not adjacent.

We first show that, under this hypothesis, there is a prime in $\pi$ which is adjacent to both $r$ and $s$.

Lemma 4.2. Assume Hypothesis 4.1. Then there exists a prime $q \in \pi$ such that $q$ is adjacent in $\Delta(G)$ to both $r$ and $s$.

Proof. If $\pi=\{q\}$, then $\Delta(G)$ is a square and the conclusion is trivial. Thus, we may assume $|\pi| \geq 2$, and we work by contradiction. Suppose there is no vertex in $\pi$ is adjacent to both $r$ and $s$. Hence, there must exist two vertices say $q_{1}, q_{2}$ in $\pi$ such that $r$ is adjacent to $q_{1}$ but is not adjacent to $q_{2}$ and $s$ is adjacent to $q_{2}$ but is not adjacent to $q_{1}$. Consider the complement of $\Delta(G)$. The induced graph by $p, r, s, q_{1}, q_{2}$ is a pentagram, which also is a cycle of length 5 , a contradiction with [1, Theorem A], which states that, when $G$ is solvable, the complement of $\Delta(G)$ has no cycles of odd length. This proves the result.

We next show that there is a nonlinear irreducible character of $P$ that extends to $G$.

Lemma 4.3. Assume Hypothesis 4.1. Then there exists a character $\theta \in \operatorname{NL}(P)$ such that $\theta$ extends to $G$.

Proof. We work by contradiction. Hence, we suppose that no character in NL( $P)$ extends to $G$. By [3, Corollary 8.16], this implies that there is no character in $\mathrm{NL}(P)$ which is invariant under $H$. 
It is not difficult to see that there will exist a character $\theta \in \mathrm{NL}(P)$ that extends to $G$ if and only if $\operatorname{NL}(G)$ contains a character $\chi$ so that $\chi(1)$ is a $p$-power. By [7, Lemma 3.4], we know that $\mathbf{C}_{P^{\prime}}(H)>1$ if and only if there exists $\chi \in \operatorname{NL}(G)$ so that $\chi(1)$ is a $p$-power. Hence, some character in $\mathrm{NL}(P)$ extends to $G$ if and only if $\mathbf{C}_{P^{\prime}}(H) \neq 1$. Since we are assuming that no character in NL $(P)$ extends to $G$, we have $\mathbf{C}_{P^{\prime}}(H)=1$. Let $U$ be a Hall $\pi$-subgroup of $G$. We will prove that $A \leq \mathbf{Z}(G)$ and $U \leq \mathbf{Z}(H)$.

By the structure of $\Delta(G)$, there exists a character $\chi_{r} \in \operatorname{Irr}(G), \chi_{r}(1)=p^{a} r^{b}$, where $a$ and $b$ are positive integers. Let $\theta \in \operatorname{Irr}(P)$ be a constituent of $\left.\left(\chi_{r}\right)\right|_{P}$. Observe that $\theta(1)_{p}=\chi_{r}(1)_{p}$, so $\theta \in \mathrm{NL}(P)$. Let $I$ be the stabilizer of $\theta$ in $G$. We know that $\theta$ is not $G$-invariant, so $I<G$. Since $|G: I|=|H: I \cap H|$ divides $\chi_{r}(1)$, we deduce that $|G: I|$ is a power of $r$ and $I \cap H$ contains a Hall$\pi \cup\{s\} \cup \rho(G)^{\prime}$-subgroup of $G$, say $B$. By [3, Corollary 12.34], we know since no prime in $\rho(G)^{\prime}$ divides a degree in $\operatorname{cd}(G)$ that $A$ is a normal abelian subgroup of $G$. It follows that $A \leq B$. By replacing $\chi_{r}$ by a conjugate, we may assume that $U \leq B$.

Now, every degree in $\operatorname{cd}(G \mid \theta)$ is divisible by $\theta(1)|G: I|$. Hence, every degree in $\operatorname{cd}(G \mid \theta)$ has the form $p^{\alpha} r^{\beta}$ for positive integers $\alpha$ and $\beta$. By Clifford's theorem, no prime in $\pi \cup\{s\}$ will divide any degree in $\operatorname{cd}(I \mid \theta)$. Applying [3, Corollary 8.16], we see that $\theta$ extends to $I$. We now apply Gallagher's theorem to see that no prime in $\pi \cup\{s\}$ divides a degree in $\operatorname{cd}(I / P)=\operatorname{cd}(I \cap H)$. In light of Itô's theorem, $B$ is abelian and normal in $I \cap H$. We conclude that $[A, U]=[A, S]=[U, S]=1$, where $S$ is some Sylow $s$-subgroup of $H$.

Similarly, if we take $\chi_{s} \in \operatorname{Irr}(G)$ with $\chi_{s}(1)=p^{c} s^{d}$, where $c$ and $d$ are positive integers, then we obtain $[A, R]=[U, R]=1$, where $R$ is some Sylow $r$-subgroup of $H$. Note that $H=A U R S$, so $A$ and $U$ are both central in $H$. It is clear that $[A, P]=1$, and we conclude that $A \leq \mathbf{Z}(G)$. Since $U$ and $A$ are central in $H$, we have $H=U A \times R S$. In particular, we obtain $H^{\prime} \leq R S$.

By Lemma 4.2, we can find a prime $q \in \pi$ that is adjacent to both $r$ and $s$. Let $G_{1}=P \rtimes(Q \times R S)$, where $Q$ is a Sylow $q$-subgroup of $H$. Then $G_{1}$ is normal in $G$ since $G^{\prime} \leq G_{1}$. It is not difficult to see that $\rho\left(G_{1}\right)=\{p, q, r, s\}$ and that there are edges in $\Delta\left(G_{1}\right)$ between $p$ and both $r$ and $s$ and between both $r$ and $s$ and $q$. Hence, $\Delta\left(G_{1}\right)$ is a square. By [7, Lemma 3.4], we know that $\mathbf{C}_{P^{\prime}}(Q R S)$ is nontrivial. Notice that $P U$ is normal in $G$. From the structure of $\Delta(G)$, we know that $\Delta(P U)$ is disconnected and satisfies the hypotheses of [4, Example 2.1] (this follows from Lemma $2.3(\mathrm{~b})$ and (h)), and hence $\left[P^{\prime}, U\right]=1$. Recall that $H=A U \times R S$ and $A \leq \mathbf{Z}(G)$. It follows that $\mathbf{C}_{P^{\prime}}(H)=\mathbf{C}_{P^{\prime}}(Q R S)$. Since $\mathbf{C}_{P^{\prime}}(Q R S)$ is nontrivial, this implies that $C_{P^{\prime}}(H)$ is nontrivial, which is a contradiction. This proves the result. 
Suppose Hypothesis 4.1. Now, we know there exists a character $\theta \in \operatorname{NL}(P)$ such that $\theta$ is extensible to $G$. Using Gallagher's theorem,

$$
\operatorname{cd}(G \mid \theta)=\{\theta(1) b \mid b \in \operatorname{cd}(G / P)\} .
$$

It follows that $\rho(G / P)=\rho(H) \subseteq\{r, s\}$, and in particular, no prime in $\pi$ divides any degree in $\operatorname{cd}(H)$. From Itô's theorem, we see that $H$ has an abelian normal Hall $\pi$-subgroup, say $Q$. Notice that either $|\rho(H)|=1$ or $\rho(H)=\{r, s\}$. Next, we consider two cases. The first case is $|\rho(H)|=1$. The second case is $\rho(H)=\{r, s\}$.

As we have seen before, if $\pi=\{q\}$, then by [7, Main Theorem], the proof of the main theorem is finished. In the following, we assume that $\pi$ contains at least two elements.

Lemma 4.4. Assume Hypothesis 4.1, $|\pi| \geq 2$, and $\rho(H)=\{s\}$. Then we have $G=H_{1} \times H_{2} \times A$, where $\Delta\left(H_{1}\right)$ is disconnected with $\rho\left(H_{1}\right)=\{p\} \cup \pi$ and $\Delta\left(H_{2}\right)$ is disconnected with $\rho\left(H_{2}\right)=\{r, s\}$.

Proof. By Itô's theorem, $H$ has an abelian, normal Hall $s^{\prime}$-subgroup, so if we write $R$ for the Sylow $r$-subgroup and $U$ for the Hall $\pi$-subgroup, then the normal Hall $s^{\prime}$-subgroup of $H$ is $A \times R \times U$. This yields $H=(A \times R \times U) S$, where $S$ is a Sylow $s$-subgroup of $H$. In particular, $P R$ is a normal subgroup of $G$.

We know there exists a character $\chi \in \operatorname{Irr}(G)$ with $\chi(1)=p^{a} r^{b}$, where $a, b$ are positive integers. Observe that $\chi_{P R}$ is irreducible by [3, Corollary 11.29]. Applying Gallagher's theorem, the only possible prime divisors of characters in $\operatorname{cd}(G / P R)$ are $p$ and $r$. Since $p$ and $r$ do not divide $|G: P R|$, we deduce that $G / P R \cong H / R$ is abelian. That is, $H^{\prime} \leq R$. We see that $[H, A U] \leq R \cap A U=1$, so $A$ and $U$ are central in $H$. This implies that $H=A \times U \times R S$. Since $A$ centralizes $P$, we have that $A$ is central in $G$. Also, since $S$ is isomorphic to a subgroup of $H / R$, we see that $S$ is abelian.

Using Lemma 4.2, we can find a prime $q \in \pi$ that is adjacent to both $r$ and $s$. Let $Q$ be a Sylow $q$-subgroup of $H$, and set $L=P \rtimes(Q R S)$. Since $G^{\prime} \leq L$, we see that $L$ is normal in $G$. Clearly, $L$ satisfies the hypotheses of [7, Lemma 3.5]. That result implies that $L=C_{P}(R) Q \times[P, R] R S$, where $C_{P}(R)$ is nonabelian. In particular, $\operatorname{Irr}\left(C_{P}(R) Q\right)$ contains characters whose degrees are divisible by $p$.

Next, we show that $[P, R, U]=1$. Suppose $t \in \pi$, and take $T$ to be a Sylow $t$-subgroup of $G$ contained in $U$. Notice that $T$ centralizes $R$ and $S$. It follows that $T$ will normalize $C_{P}(R) Q$ and $[P, R] R S$. If $[P, R, T] \neq 1$, then there exists a character in $\operatorname{Irr}([P, R])$ that will not be $T$-invariant. It then follows that $\operatorname{Irr}([P, R] R S)$ contains a character $\gamma$ that is not $T$-invariant. Let $\psi \in \operatorname{Irr}\left(C_{P}(R) Q\right)$ so that $p$ divides $\psi(1)$. Let $\chi \in \operatorname{Irr}(G \mid \psi \times \gamma)$. It follows that $\chi(1)$ is divisible by $p t$, a contradiction of the structure of $\Delta(G)$, where $p$ is not adjacent to any 
prime in $\pi$. We conclude that every Sylow subgroup of $U$ centralizes $[P, R]$, so $U$ centralizes $[P, R]$. Now, let $H_{1}=C_{P}(R) U$ and $H_{2}=[P, R] R S$. Then we have $G=L U A=H_{1} \times H_{2} \times A$.

We note that, for the record, the subgroup $H_{1}$ in Lemma 4.4 satisfies the hypotheses of [4, Example 2.1] (by Lemma 2.3(b) and (h)) and $H_{2}$ satisfies the hypotheses of [4, Example 2.4].

We now consider the second case $\rho(H)=\{r, s\}$. We note that $M$ in this result satisfies the hypotheses of [4, Example 2.1] and $N$ can satisfy the hypotheses any of [4, Examples 2.1-2.5 but not Example 2.6].

Lemma 4.5. Assume Hypothesis 4.1, and let $\rho(H)=\{r, s\}$. Then $G=M \times N$, where $\Delta(M)$ and $\Delta(N)$ are disconnected with $P \leq M$ and $\rho(N)=\{r, s\}$.

Proof. We have that $\rho(H)=\{r, s\}$. Since $H \cong G / P$, we see that $\Delta(H)$ is a subgraph of $\Delta(G)$. In particular, $\Delta(H)$ is disconnected. If $H$ satisfies the hypotheses of [4, Example 2.6], then $|\rho(H)| \geq 3$ by Lemma 2.3 (h), so $H$ cannot satisfy the hypotheses of [4, Example 2.6]. From Itô's theorem, $H$ has an abelian normal Hall $\pi$-subgroup, say $U$. It follows that $P U$ is normal in $G$. We note that $\Delta(P U)$ has two connected components $\{p\}$ and $\pi$. Since $P U$ has Fitting height 2, we know via Lemma 2.3 (i) that $P U$ satisfies the hypotheses of [4, Example 2.1].

Suppose for now that $H$ does not satisfy the hypotheses of [4, Example 2.4]. Then $H$ has a central Hall $\rho(H)$-complement. This implies $U A=U \times A \leq \mathbf{Z}(H)$, in particular, $A \leq \mathbf{Z}(G)$. Since $H$ has a central Hall $\{r, s\}$-complement, $H$ has a normal Hall $\{r, s\}$-subgroup, say $H_{1}$. We have $H=A U \times H_{1}$. It is clear that $\Delta\left(H_{1}\right)$ is disconnected.

In light of Lemma 4.2, there is a prime $q \in \pi$ that is adjacent to both $r$ and $s$. Next, we consider the group $G_{1}=P Q H_{1}$, where $Q$ is a Sylow $q$-subgroup of $H$ for some prime $q \in \pi$. Observe that $G_{1}$ is normal in $G$. It is not difficult to see that $\Delta\left(G_{1}\right)$ is a square. Applying [7, Main Theorem], $G_{1}=P Q \times H_{1}$. We conclude that $G=P U A \times H_{1}$. Letting $M=P U A$ and $N=H_{1}$, we obtain the conclusion in this case.

We now consider the case where $H$ satisfies the hypotheses of [4, Example 2.4]. Let $H=V L$ be the semi-direct product of $V$ with $L$ as given by Lemma $2.3(\mathrm{~g})$. Let $t$ be the prime so that $V$ is a $t$-group. Let $K=\mathbf{F}(L)$ and $Z=\mathbf{C}_{L}(V)$. Since the connected components of $\Delta(H)$ are $\{r\}$ and $\{s\}$, the index $|K / Z|$ is a prime power, say $r^{a}$; and similarly, $|H: V K|$ is a power of $s$. Observe that $V$ is an elementary abelian $t$-group.

First, we assume that $t$ is not in $\pi$. It is clear that $U \leq \mathbf{Z}(H)$. Let $H_{1}$ be a Hall $\pi$-complement of $H$, so $H=H_{1} \times U$. Let $Q$ be a Sylow $q$-subgroup of $U$. Write $G_{1}=P Q H_{1}$; then $G_{1} \triangleleft G$, and $\Delta\left(G_{1}\right)$ is a square. By [7, Main Theorem], we 
know that $G_{1}=P Q \times H_{1}$, and hence $G=P U \times H_{1}$. Let $M=P U, N=H_{1}$, and we are done with this situation.

Now, suppose $t \in \pi$; without loss of generality, we may assume that $t=q$. Clearly, $A \leq \mathbf{Z}(G)$. From Lemma 2.3 (c), we see that $Q=V \times(Q \cap \mathbf{Z}(H))$. Write $Q_{1}=V$ and $Q_{2}=Q \cap \mathbf{Z}(H)$. Let $W$ be the Hall $q$-complement of $U$; then $W \leq \mathbf{Z}(H)$. Let $R$ be a Sylow $r$-subgroup of $H$. By Lemma $2.3(\mathrm{~g})$, we have $Q_{1}=[Q, H] \geq[Q, \mathbf{F}(L)]=[Q, R]$. Since $[Q, R] \triangleleft H$ and $Q_{1}$ is irreducible under the action of $R$, we have $Q_{1}=[Q, R]$. Write $G_{1}=P\left(Q_{1} R S\right)$, where $S$ is some Sylow $s$-subgroup of $H$ that normalizes $R$. Since $A$ and $U$ both normalize $G_{1}$, it follows that $G_{1} \triangleleft G$.

We claim that $\left[P, Q_{1}\right]=1$. If not, observe that $\rho\left(G_{1}\right)=\{p, q, r, s\}$. It follows that $\Delta\left(G_{1}\right)$ is a square. By [7, Main Theorem], we know $G_{1}$ is a direct product of subgroups, and this violates the fact that $Q_{1}$ is irreducible under $R$. Thus, $Q_{1}$ centralizes $P$. In particular, $Q_{1}$ is normal in $G_{1}$. We see that $\Delta\left(G_{1} / P^{\prime}\right)$ has two connected components $\{r\}$ and $\{s\}$.

Notice that $\Delta\left(Q_{1} R S\right)$ is disconnected and that $Q_{1} R S$ satisfies the hypotheses of [4, Example 2.4] (this follows from Lemma 2.3 (c) and $q \notin \rho\left(Q_{1} R S\right)$ ). Also, $Q_{1} R S$ is isomorphic to a factor group of $G_{1} / P^{\prime}$. We deduce that $\Delta\left(G_{1} / P^{\prime}\right)$ is disconnected and that $G^{\prime} / P$ satisfies the hypotheses of one of [4, Example 2.4, 2.5 or 2.6]. Since the degree graphs of groups satisfying the hypotheses of [4, Example 2.6] have at least 3 vertices (see Lemma 2.3(h)) and the Fitting subgroups of groups satisfying the hypotheses of [4, Example 2.5] are nonabelian (see Lemma $2.3(\mathrm{~d})$ ), we see that $G_{1} / P^{\prime}$ cannot satisfy the hypotheses of [4, Example 2.5 or 2.6]. Hence, $G_{1} / P^{\prime}$ satisfies the hypotheses of [4, Example 2.4]. We deduce that $P / P^{\prime} \leq \mathbf{Z}\left(G_{1} / P^{\prime}\right)$. In particular, $Q_{1} R S$ will centralize $P$, so $G_{1}=$ $P \times Q_{1} R S$. We conclude that $G=P Q_{2} W A \times Q_{1} R S$. Taking $M=P Q_{2} W A$ and $N=Q_{1} R S$, we have the result in this situation.

We are ready to give the proof of Theorem 1.3.

Proof of Theorem 1.3. If $\pi=\{q\}$, then, by [7, Main Theorem], we are done. By Lemma 4.3, there exists a character $\theta \in \mathrm{NL}(P)$ such that $\theta$ is extendable to $G$. And hence $|\rho(H)|=1$ or $\rho(H)=\{r, s\}$. When $|\rho(H)|=1$, without loss of generality, we may assume $\rho(H)=\{s\}$, and we finish the proof using Lemma 4.4. For the second case, we use Lemma 4.5 to obtain the result.

Acknowledgments. The second author would like to give her sincere thanks to the Department of Mathematical Sciences of Kent State University (KSU) for its hospitality and much appreciation to her friend Mark J. Miller for his support during her visit at KSU. Last but not least, the authors would like to thank the referee for the careful reading of this paper. 


\section{Bibliography}

[1] Z. Akhlaghi, C. Casolo, S. Dolfi, K. Khedri and E. Pacifici, On the character degree graph of solvable groups, Proc. Amer. Math. Soc. 146 (2018), no. 4, 1505-1513.

[2] C. Casolo, S. Dolfi, E. Pacifici and L. Sanus, Groups whose character degree graph has diameter three, Israel J. Math. 215 (2016), no. 2, 523-558.

[3] I. M. Isaacs, Character Theory of Finite Groups, Pure Appl. Math. 69, Academic Press New York, 1976,

[4] M. L. Lewis, Solvable groups whose degree graphs have two connected components, J. Group Theory 4 (2001), no. 3, 255-275.

[5] M.L. Lewis, Solvable groups with character degree graphs having 5 vertices and diameter 3, Comm. Algebra 30 (2002), no. 11, 5485-5503.

[6] M. L. Lewis, An overview of graphs associated with character degrees and conjugacy class sizes in finite groups, Rocky Mountain J. Math. 38 (2008), no. 1, 175-211.

[7] M.L. Lewis and Q. Meng, Square character degree graphs yield direct products, J. Algebra 349 (2012), 185-200.

[8] M.L. Lewis and Q. Meng, Solvable groups whose prime divisor character degree graphs are 1-connected, Monatsh. Math. 1 (2019), DOI 10.1007/s00605-01901276-8.

[9] O. Manz, R. Staszewski and W. Willems, On the number of components of a graph related to character degrees, Proc. Amer. Math. Soc. 103 (1988), no. 1, 31-37.

[10] C. P. Morresi Zuccari, Character degree graphs with no complete vertices, J. Algebra 353 (2012), 22-30.

[11] C. P. Morresi Zuccari, Fitting height and diameter of character degree graphs, Comm. Algebra 41 (2013), no. 8, 2869-2878.

[12] P. P. Pálfy, On the character degree graph of solvable groups. I. Three primes, Period. Math. Hungar. 36 (1998), no. 1, 61-65.

[13] C. B. Sass, Character degree graphs of solvable groups with diameter three, J. Group Theory 19 (2016), no. 6, 1097-1127.

Received February 7, 2019; revised August 28, 2019.

\section{Author information}

Mark L. Lewis, Department of Mathematical Sciences,

Kent State University, Kent, Ohio 44242, USA.

E-mail: lewis@math. kent. edu

Qingyun Meng, College of Sciences, Henan University of Technology,

Zhengzhou, 450001, P. R. China.

E-mail: mqyzhy@126. com 